\section{Eradication of a Multidrug-Resistant, Carbapenemase-Producing Klebsiella pneumoniae Isolate Following Oral and Intra-rectal Therapy With a Custom Made, Lytic Bacteriophage Preparation}

\begin{abstract}
Mario Corbellino, ${ }^{1, \oplus}$ Nicolas Kieffer, ${ }^{2}$ Mzia Kutateladze, ${ }^{3}$ Nana Balarjishvili, ${ }^{3}$ Lika Leshkasheli, ${ }^{3}$ Lia Askilashvili, ${ }^{3}$ George Tsertsvadze, ${ }^{3}$ Sara Giordana Rimoldi, Deia Nizharadze, ${ }^{5}$ Naomi Hoyle, ${ }^{5}$ Lia Nadareishvili, ${ }^{5}$ Spinello Antinori, ${ }^{1,6}$ Cristina Pagani, ${ }^{4}$ Daniele Giuseppe Scorza, ${ }^{4}$ Ai Ling Loredana Romanò, ${ }^{7}$ Sandro Ardizzone, ${ }^{6,8}$ Piergiorgio Danelli, ${ }^{6,9}$ Maria Rita Gismondo, ${ }^{4,6}$ Massimo Galli, ${ }^{1,6}$ Patrice Nordmann, ${ }^{2}$ and Laurent Poirel ${ }^{2}$

${ }^{1}$ III Division of Infectious Diseases, Azienda Socio Sanitaria Territoriale (ASST) Fatebenefratelli Sacco, Milano, Italy: ${ }^{2}$ Medical and Molecular Microbiology, Faculty of Science and Medicine, Institut National de la Santé et de la Recherche Médicale (INSERM) European Unit (IAME, France), University of Fribourg, Fribourg, Switzerland; ${ }^{3} G$. Eliava Institute of Bacteriophages, Microbiology and Virology, Tbilisi, Georgia; ${ }^{4}$ Division of Microbiology, ASST FatebenefratelliSacco, Milano, Italy; ${ }^{5}$ Eliava Phage Therapy Center LLC, Tbilisi, Georgia; ${ }^{6}$ Luigi Sacco Department of Clinical and Biomedical Sciences, University of Milano, Milano, Italy; ${ }^{7}$ Division of Urology, ASST Fatebenefratelli - Sacco, Milano, Italy; ${ }^{8}$ Division of Gastroenterology, ASST Fatebenefratelli - Sacco, Milano, Italy; and ${ }^{9}$ Division of General Surgery, ASST Fatebenefratelli - Sacco, Milano, Italy
\end{abstract}

In July 2017, a patient presented colonization with a multidrugresistant, carbapenemase (KPC-3)-producing Klebsiella pneumoniae isolate. A custom-made, lytic bacteriophage preparation was administered to the patient in December 2017, with subsequent eradication of the microorganism and without adverse effects.

Keywords. multidrug-resistant Klebsiella pneumoniae; antibiotic resistance; bacteriophage; personalized phage therapy; selective decolonization.

Infections caused by multidrug-resistant (MDR), carbapenemase-producing (CP) Klebsiella pneumoniae (Kp) are responsible for a rapidly growing burden of disease worldwide [1]. Among hospitalized adults, asymptomatic carriage of MDR Enterobacterales (ie, colonization) precedes, and significantly augments, the risk of developing infections caused by these microorganisms [2]. In addition, colonization may be protracted, persisting in 39\% of patients after 12 months of observation, with a mean time for spontaneous decolonization after hospital discharge of 387 days [3].

Gut colonization by MDR Enterobacterales has also been established in healthy travelers returning from endemic areas of

Correspondence: M. Corbellino, III Division of Infectious Diseases, ASST Fatebenefratelli Sacco, Milano, Italy (mario.corbellino@unimi.it). antimicrobial resistance who were without a history of contact with medical care while abroad, with a demonstrated length of carriage of at least 6 months [4-6].

Besides the use of antibiotics, alternatives are urgently needed to significantly contribute to the eradication of MDR bacteria in general, and CP Gram-negative bacteria in particular. Among those alternative strategies, the use of phage therapy is currently being reconsidered for treating corresponding infections. Custom-made bacteriophage therapy (BT) consists of the clinical use of viruses that have been preliminarily selected in vitro for their specific and strictly lytic activity against a bacterial pathogen isolated in culture, with the aim of treating the infection sustained by the pathogen [7]. Several examples of the successful treatment of infections caused by MDR bacteria have been reported [7-9]. However, to the best of our knowledge, although it has been suggested [10], no gut decolonization strategy using bacteriophages has been reported to date.

We describe herein a patient for whom custom-made BT was employed to clear a long-standing colonization by a repeatedly invasive MDR and CP Kp isolate. The sequence of phage $\mathrm{vB}_{-}$ KpnM_GF has been deposited in the Genbank databases under accession number MK421971.

\section{CASE REPORT}

In July 2017, a 57-year-old patient with a remote diagnosis of Crohn's disease, which had been in clinical remission since 2015 , came to our attention for the multi-site colonization (ie, the gastrointestinal tract, the urinary tract, and a permanent external invasive device) of an MDR Kp strain with a 1-month duration.

Because of recurrent episodes of obstructive nephrolithiasis and urinary tract infections (UTIs) that occurred over a period of 3 years and were complicated by massive fibrosis of the bladder (residual volume: $25 \mathrm{ml}$ ), the patient had undergone right nephrectomy and radical cystectomy 5 months earlier (in February 2017), with the creation of a left cutaneous ureterostomy and positioning of a ureteral stent. The latter could not be removed because of rapidly ensuing hydronephrosis of the solitary kidney. The patient did not take medications and had Stage III chronic renal failure (creatinine clearance, $30 \mathrm{ml}$ / $\min )$.

Rectal swab screening allowed the recovery of an MDR Kp isolate belonging to the Sequence Type ST307. That isolate was resistant to all ß-lactams, including carbapenems, and produced the KPC-3 carbapenemase. It remained susceptible only to ceftazidime-avibactam (CZA), amikacin, fosfomycin, and tigecycline (see Supplementary Material). 
In August 2017, the patient was admitted with sepsis to the hospital in Milan. Owing to her colonization history with a CP $K p$, she was empirically treated with CZA, with rapid resolution of the infection. Blood cultures, which had been confirmed positive for the MDR $K p$ at admission, were negative at 72 hours after the initiation of antimicrobial therapy. However, a culture of the proximal tip of the ureteral stent, which was replaced after 12 days of therapy with CZA, showed persistence of the microorganism. At 1 week after discharge, a urine culture, as well as a rectal swab of the patient, again turned positive for the MDR Kp, which nevertheless remained susceptible to CZA (see Table 1).

Given the patient's high risk of recurrent, invasive infections, sustained by the persistently colonizing MDR microorganism and the limited antibiotic armamentarium for treatment, in midOctober 2017, $5 \mathrm{Kp}$ isolates that had been obtained at different time points from the urine $(n=3)$, rectal swab $(n=1)$, and ureteral stent $(n=1)$ of the patient were sent to the Georgi Eliava Institute of Bacteriophages in Tbilisi, Georgia, in order to obtain a custom-made, lytic bacteriophage preparation that would be active against the microorganism (see Supplementary Material). During the 9-week period of custom-made phage preparation, the patient actually experienced 2 additional UTIs, sustained by the MDR Kp. However, in both circumstances, the MDR Kp readily reappeared in the cultures of the rectal swab and the urine of the patient a few days after interruption of the antimicrobial therapy (see Table 1).

In December, the patient travelled to Tbilisi to collect the phage preparation and be instructed on its proper use. On this occasion, the physicians of the Eliava Phage Therapy Center obtained written informed consent to the treatment from the patient.

However, 2 days later, and before starting BT, the patient developed a fever and chills and immediately returned to Milan, where she was directly hospitalized upon arrival.

Urine and blood cultures were collected and she was empirically given CZA, with a prompt recovery. Her urine cultures were positive for the same CZA-susceptible $K p$, as assessed by pulsed-field gel electrophoresis (detailed in the Supplementary Material), whereas her blood cultures remained negative. The ureteral stent was replaced on the fourth day of antibiotic therapy and a culture of its proximal tip still tested positive for the CP Kp. CZA was interrupted after 5 days of treatment and the patient was discharged the following day, while asymptomatic.

On 22 December 2017, 3 days after discharge, the patient started a 3-week cycle of BT, given by the oral and intra-rectal routes (see Supplementary Material).

Since BT was given on an outpatient basis, we did not seek approval by the Ethics Committee of Luigi Sacco Hospital before treatment initiation.

The treatment was well tolerated and the patient did not experience adverse effects (see Supplementary Material).

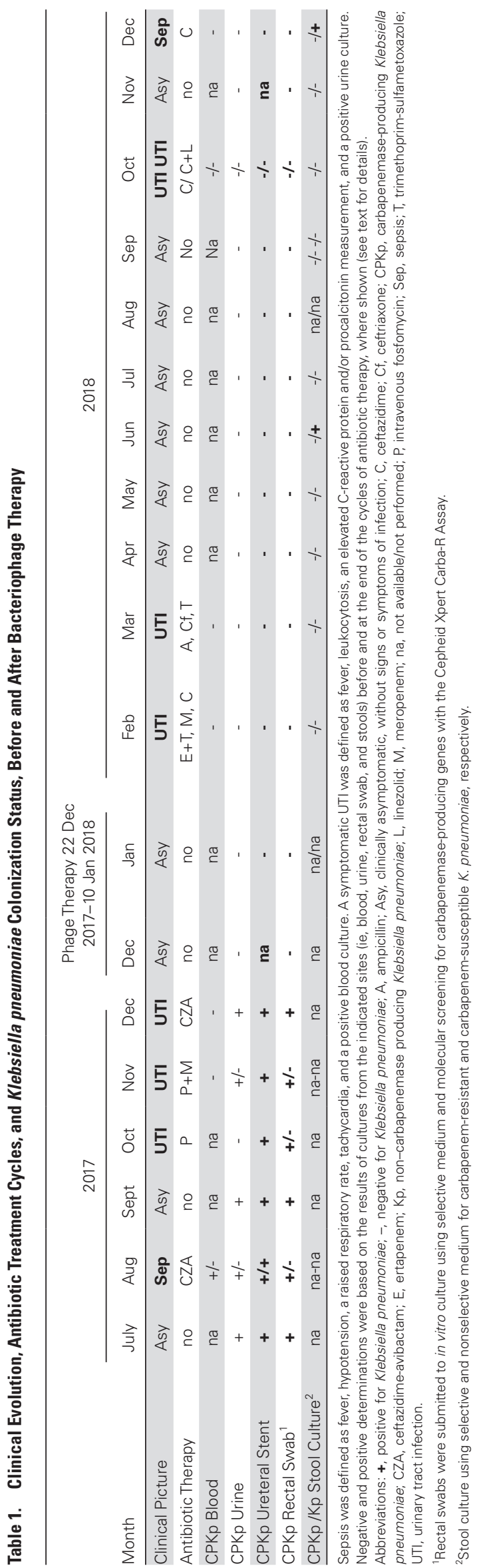


Remarkably, 15 days after the beginning of BT, the ureteral stent was replaced and, for the first time, a culture of its proximal end did not yield any $K p$.

From then onwards, and until the time of writing, all attempts to isolate MDR Kp by growth on selective media from the patient's stools, rectal swabs, urine, and the ureteral stents have been unsuccessful.

Moreover, molecular screening of the patient's rectal swabs for the presence of carbapenemase genes, started as early as 5 days after the inception of BT and performed every 7 to 14 days until December 2018, using the Cepheid Xpert Carba-R Kit, was always negative.

In addition, the isolation of non-MDR $K p$ from the patient's stools and rectal swabs by culture on nonselective media remained unsuccessful on all occasions, except for 2 temporally distant events (ie, in June and December 2018). In those latter cases, $K p$ isolates were recovered but they were susceptible to most antibiotics and were genetically unrelated to the original MDR strain, as they belonged to ST1109 (June) and ST247 (December).

\section{DISCussion}

We show herein that long-standing, multi-site colonization by an MDR Kp strain in a patient with a solitary kidney, a cutaneous ureterostomy, and a permanent ureteral stent resolved after a 3-week course of personalized BT given by both oral and intra-rectal routes. The same result was not achieved despite 2 cycles of antibiotic treatment of 14 and 5 days, respectively, with a CZA regimen towards which the MDR Kp isolates remained susceptible.

Following BT, the patient developed 4 distinct episodes of complicated UTIs and a second episode of sepsis (see Table 1 and Supplementary Material). Notwithstanding the protracted, antibiotic-driven selective pressure on the patient's microbiota and the repeated hospitalizations, we did not observe a reappearance of the MDR Kp.

This case report presents several limitations. First of all, following BT, we failed to detect the MDR Kp isolate (by culture) and the $b l a_{\mathrm{KPC}-3}$ carbapenemase gene (by direct molecular testing) from the stools and rectal surveillance swabs of the patient, but a lack of sensitivity of detection with both approaches could be argued. Nevertheless, excellent sensitivity of the Cepheid Xpert Carba-R assay has been proven [11]. Hence, the unsuccessful and repeated attempts over a period of more than 1 year suggest the bona fide eradication of the MDR pathogen.

Second, we did not measure the concentration of CZA in the urine or blood of the patient when BT was initiated (ie, 72 hours after the last administration of the antibiotic). Given the drug's half-life and the patient's reduced creatinine clearance, it is probable that CZA was still present in the urine at the time of BT initiation. For this reason, we cannot exclude an eventual role of phage-antibiotic synergy in the eradication of the microorganism [12].

Finally, albeit still poorly understood, the phenomenon of spontaneous decolonization in carriers of MDR pathogens has been well documented [4-6]. Thus, and because this is a single case report, we cannot draw any conclusion on the causal role of $\mathrm{BT}$ in the eradication of the patient's MDR Kp.

\section{CONCLUSION}

Given the magnitude of the threat posed by antibiotic resistance worldwide, we believe that controlled studies are urgently needed to demonstrate the safety and efficacy of custom-made phage therapy for the selective decolonization of individuals who are carriers of MDR bacterial pathogens. A personalized phage therapy approach might constitute an interesting alternative to antibiotic usage and participate in the global effort to minimize the risk of selection of pan drug-resistant strains.

\section{Supplementary Data}

Supplementary materials are available at Clinical Infectious Diseases online. Consisting of data provided by the authors to benefit the reader, the posted materials are not copyedited and are the sole responsibility of the authors, so questions or comments should be addressed to the corresponding author.

\section{Notes}

Author contributions. M. C. had contact with the G. Eliava Institute of Bacteriophages and the G. Eliava Phage Therapy Center. N. K., L. P., and P. N. conducted the molecular characterization and host-range determination of the bacteriophage. M. K., N. B., L. L., and G. T. managed the bacteriophage selection and adaption procedures, production, ultrastructural characterization, and host-range determination. S. G. R., C. P., and M. R. G. managed the isolation, molecular typing and antimicrobial susceptibility determination, and molecular screening for carbapenemase genes of the multidrug-resistant Klebsiella pneumoniae. D. N., N. H., and L. N. determined the bacteriophage therapy dosage, routes of administration, and duration of treatment. M. C., S. A., D. G. S., A. L. L. R., S. A., P. D., and M. G. provided clinical care for the patient. M. C., L. P., and M. K. wrote the manuscript. All authors read and approved the final manuscript.

Acknowledgments. The authors thank their exceptional patient for her trust.

Financial support. This work was supported by the Laboratoire Européen Associè à l' Institut National de la Santé et de la Recherche Mèdicale, Emerging Antibiotic Resistance in Gram-negative bacteria, the Univeristy of Fribourg; the "Eliava Foundation" in Tbilisi; and the Associazione Nazionale per Lotta all'AIDS, Sezione Lombarda, Milano.

Potential conflicts of interest. N. B. and L. A. received payment from the patient for the preparation of the phage through the Eliava Institute of Bacteriophages, Microbiology, and Virology. L. L. received personal fees from the Eliava Institute of Bacteriophages, Microbiology, and Virology during the conduct of the study. All other authors report no potential conflicts. All authors have submitted the ICMJE Form for Disclosure of Potential Conflicts of Interest. Conflicts that the editors consider relevant to the content of the manuscript have been disclosed.

\section{References}

1. Cassini A, Högberg LD, Plachouras D, et al. Attributable deaths and disabilityadjusted life-years caused by infections with antibiotic-resistant bacteria in the EU and the European Economic Area in 2015: a population-level modeling analysis. Lancet Inf Dis 2019; 19:56-66. 
2. Tischendorf J, Almeida de Avila R, Safdar NS. Risk of infection following colonization with carbapenem-resistant Enterobacteriaceae: a systematic review. Am J Inf Contr 2016; 44:539-43.

3. Zimmerman FS, Assous MV, Bdolah-Abra T, Lachish T, Yinnon AM, WienerWell Y. Duration of carriage of carbapenem-resistant Enterobacteriaceae following hospital discharge. Am J Inf Contr 2013; 41:190-94.

4. Armand-Lefèvre L, Andremont A, Ruppé E. Travel and acquisition of multidrugresistant Enterobacteriaceae. Med Maladies Infect 2018; 48:431-41.

5. Arcilla MS, van Hattem JM, Haverkate MR, et al. Import and spread of extended spectrum beta-lactamase producing Enterobacteriaceae by international travellers (COMBAT study): a prospective, multicentre cohort study. Lancet Infect Dis 2017; 78-85. doi:10.1016/S1473-3099(16)30319X.

6. Van Hattem JM, Arcilla MS, Bootsma MCJ, et al. Prolonged carriage and potential onward transmission of carbapenemase-producing Enterobacteriaceae in Dutch travelers. Future Microbiology 2016; 11:857-64.

7. Altamirano FLG, Barr JJ. Phage therapy in the post-antibiotic era. Clin Microbiol Rev 2019; 32:e00066-18.
8. Schooley RT, Biswas B, Gill JJ, et al. Development and use of personalized bacteriophage-based therapeutic cocktails to treat a patient with a disseminated resistant Acinetobacter baumannii infection. Antimicrob Agents Chemother 2017; 61:e00954-17.

9. Ferry T, Boucher F, Fevre C, et al. Innovations for the treatment of a complex bone and joint infection due to XDR Pseudomonas aeruginosa including a local application of a selected cocktail of bacteriophages. J Antimicrob Chemother 2018; 73:2901-03.

10. Tacconelli E, Mazzaferri F, de Smet AM, et al. ESCMID-EUCIC clinical guidelines on decolonization of multidrug-resistant Gram-negative bacteria carriers. Clin Microbiol and Inf 2019; 25:807-17.

11. Tato M, Ruiz-Garbajosa P, Traczewski M, et al. Multisite evaluation of Cepheid Xpert Carba- $\mathrm{R}$ assay for detection of carbapenemase-producing organisms in rectal swabs. J Clin Microbiol 2016; 54:1814-19.

12. Oechslin F, Piccardi P, Mancini S, et al. Synergistic interaction between phage therapy and antibiotics clears Pseudomonas aeruginosa infection in endocarditis and reduces virulence. J Inf Dis 2017; 215:703-12. 